\title{
Poser des questions ce n'est jamais un scandale ! Interview politique, question contrediscursive médiée et polémique
}

\author{
Aleksandra Nowakowska, Jacques Bres \\ UMR 5267 Praxiling Université Montpellier III \\ aleksandra.nowakowska@univ-montp3.fr \\ jacques.bres@univ-montp3.fr
}

\begin{abstract}
Résumé
Partant du constat que les questions des journalistes dans l'interview politique brève se signalent fréquemment par les deux traits de la contrediscursivité et de la médiation énonciative, notre contribution décrit dans un premier temps l'association de ces deux éléments, en fournit ensuite une explication en relation avec les représentations du discours politique et avec les attentes médiatiques, avant d'en analyser le potentiel polémique : ce qui fait que ce type de question, structuralement hors polémique, peut cependant y conduire.
\end{abstract}

Mots-clés : question, interview, discours politique, contrediscursivité, médiation énonciative, polémique

Les médias en tant qu'outils de diffusion de l'information rapportent les activités de la sphère politique, notamment les activités discursives ; ils ont une affection toute particulière pour les petites phrases susceptibles de susciter la polémique. Mais ils ne se contentent pas de transmettre les discours des politiques, ils participent aussi activement à leur production, et s'inscrivent notamment dans la dimension conflictuelle qui est une des composantes de ce type de discours. Par différents genres, les médias relancent le débat, voire le suscitent, jettent de l'huile sur le feu de la polémique, en attisent les braises...

Nous nous intéresserons dans cette communication à un genre médiatique particulier, l'interview politique brève. Ce genre est en prise avec l'actualité immédiate, il sollicite un discours d'opinion sur l'actualité ou, plus souvent, sur les commentaires suscités par celle-ci, ce qui oblige le journaliste à bien cibler les questions, en privilégiant quasi exclusivement les thèmes qui font débat, afin de respecter le format temporel inférieur à 10 minutes. Nous analyserons plus particulièrement un type de question qui structure ce genre, et qui, à notre connaissance, n'a pas fait l'objet d'étude spécifique, et que nous proposons de nommer question contrediscursive médiée (désormais QCM), en ce qu'elle associe deux traits : (i) elle manifeste une médiation énonciative, à savoir qu'elle se présente comme rapportant un propos tenu antérieurement par un autre locuteur, qui appartient le plus souvent à la sphère politique ou syndicale; (ii) ce propos est contrediscursif - on dira également intrusif, ou infirmatif - : il contredit l'image positive que l'interviewé, par son discours, donne de lui- 
même ou du parti auquel il appartient, et recèle en cela un fort potentiel polémique. Ces deux dimensions tendent à s’articuler de la sorte : la médiation énonciative offre au journaliste un écran énonciatif protecteur qui lui permet d'interroger l'invité politique sur des points sensibles, de poser des questions embarrassantes, voire offensantes, sans s'exposer directement et ouvertement à ses contre-attaques. L'interviewé de son côté doit contourner le piège que pose la QCM, tout en accomplissant son devoir de réponse supposé par le contrat de communication, ce qu'il tente de faire en usant de différentes stratégies discursives, qui lui permettent d'éviter que l'interaction ne tourne à l'affrontement, qu'une polémique éclate, même si cela arrive parfois.

Notre contribution décrit dans un premier temps ce type de question, notamment la façon dont elle associe les deux ingrédients de la médiation et de la contrediscursivité. Elle propose ensuite une explication de cette forte présence, en la mettant en relation avec le genre discursif de l'interview politique dans une société d’hypermédiatisation. Dans un troisième temps, on s’interroge sur les liens possibles entre QCM et polémique.

\section{Interview politique et question contrediscursive médiée : description}

Les études en analyse des conversations et en analyse des médias ont proposé des descriptions fines du genre discursif interview politique, notamment de l'asymétrie des rôles interactifs des partenaires, que nous pourrions résumer drastiquement ainsi : le journaliste pose des questions, l'homme /la femme politique répond aux questions qu'on lui pose...

Notre corpus se compose de 8 interviews télévisées brèves ${ }^{1}$ ( 8 minutes environ chacune, relevées dans les émissions quotidiennes Les quatre vérités (France 2), RTL Matin et dans des journaux télévisés, septembre 2008 - mars 2010.).

La présente recherche a pour point de départ le constat de la fréquence de questions comme :

Interview de J-F. Copé, président du groupe UMP à l'Assemblée, par O. Galzi, Les quatre vérités, France2, 9 décembre $2008^{2}$

(1) OG29 - Xavier Bertrand va prendre la tête de l'UMP / vous êtes chef de groupe UMP à l'assemblée / on dit de lui que c'est votre meilleur ennemi // c'est vrai ?

que nous analysons comme structurée sur le module à quatre éléments suivant :

(a) thème général : Xavier Bertrand va prendre la tête de l'UMP / vous êtes chef de groupe UMP à l'assemblée

(b) médiation énonciative : on dit de lui que

\footnotetext{
${ }^{1}$ Format actuellement en fort développement dans les médias français. Ce type d'interview présente des caractéristiques formelles propres, notamment par rapport à l'interview présidentielle (Clayman \& Heritage, 2002a et b) ou au débat politique (Burger M. 2004, Burger \& Martel 2005, Constantin de Chanay et KerbratOrecchioni 2007).

${ }^{2}$ Nous utilisons les conventions de transcriptions minimales suivantes : chaque tour de parole est identifié par les initiales des prénom et nom du locuteur ; le signe : note un allongement vocalique ; le signe /, une pause.
} 
(c) jugement contrediscursif : c'est votre meilleur ennemi

(d) interrogation sur ce jugement : c'est vrai ?

Les éléments (a) et (d) sont facultatifs ; (b) et (c) sont obligatoires en ce qu'ils définissent les QCM. Nous présenterons successivement la dimension contrediscursive, le fonctionnement de la médiation énonciative, puis la place des questions de ce type.

1. La dimension contrediscursive est réalisée par l'élément (c), qui verbalise un jugement critique visant l'action politique de l'interviewé (2), ou celle du groupe auquel il appartient (1, 3) :

Interview de X. Bertrand, ministre, par R. Sicard, Les quatre vérités, France2, 2 décembre 2008

(2) RS13 - (a) sur le désintérêt de cette élection / (b) Laurence Parisot la présidente de MDF a une explication / elle dit que (c) le responsable c'est vous / parce que la campagne a été organisée dans la plus grande pagaille

Interview de L. Chatel, ministre, par J.-M. Apathie, RTL Matin, 29 juin 2009

(3) JMA1 - (a) emprunt Sarkozy vous en avez parlé hier à Matignon où le gouvernement était réuni au grand complet (i) /(b) question du journal Libération à la Une ce matin (JMA montre la Une de Libération à L. Chatel) (c) «Emprunt Sarkozy : La France est-elle en faillite ? »

La critique s'alourdit lorsqu'elle prend la forme d'une attaque ad hominem portant sur les compétences de l’interviewé (4) ; ou pire, sur sa moralité (5) :

Interview de L. Chatel, ministre, par J.-M. Apathie, RTL Matin, 29 juin 2009

(4) JMA15 - Gérard Aschieri secrétaire général de la FSU à votre propos / puisque vous êtes ministre de l'éducation depuis mardi soir / «c'est quelqu'un » / il parle de vous/ « qui jusqu'ici n’a jamais manifesté d'intérêt pour l'éducation nationale »

Interview de B. Tapie, ancien ministre, par J.-M. Apathie, RTL Matin, 9 septembre 2008

(5) JMA35 - François Hollande premier secrétaire du parti socialiste « Chacun connaît les relations de Bernard Tapie à Nicolas Sarkozy il faut savoir si cette relation a pu jouer pour obtenir la sentence arbitrale $\mathrm{xx} »$

Ces questions sont en relation fortement contrediscursive avec le discours de l'homme politique qui, en vertu du principe de valorisation du même / dévalorisation de l'autre, tend à tenir des propos positifs sur son action politique personnelle, comme sur celle de son groupe d’appartenance, et plus généralement sur lui-même.

2. Le jugement critique se présente non comme énoncé directement par le journaliste mais comme rapporté, en discours direct ou indirect, à partir d'une autre source énonciative. La médiation énonciative s'articule à la contrediscursivité de différentes manières. Fréquemment, les QCM, de façon quelque peu abrupte, ne prennent pas la peine de verbaliser l'interrogation sur le jugement (élément (d)), comme en (2-5). Cette façon ex abrupto est accrue lorsque le verbe du discours citant fait l'objet d'une ellipse, la question ne présentant plus que la succession : nom de l'énonciateur + propos rapporté : 
Interview de L. Chatel, ministre, par J.-M. Apathie, RTL Matin, 29 juin 2009

(6) JMA29 - Gérard Aschieri de nouveau / « je suis interpellé par le fait que Luc Chatel reste porteparole du gouvernement / c’est à ma connaissance la première fois pour un ministre de l'éducation »

Ces effacements concourent à durcir la rugosité du propos rapporté, qui se voit presque comme jeté à la face de l’interviewé.

L'interaction de la médiation avec la charge contrediscursive du jugement peut être saisie également aux niveaux (i) des énonciateurs convoqués, et (ii) de la forme conversationnelle qu'elle prend parfois.

(i) Le journaliste se contente rarement de mettre le propos rapporté au compte d'une rumeur indéfinie ( «on dit », (1)). Il choisit plutôt, comme énonciateur du propos critique rapporté, un opposant - syndical (4), (6) ou politique (5), (7) - de l’interviewé, dont la relation d'antagonisme se voit parfois explicitée par une catégorisation comme adversaire en (7) :

Interview de B. Tapie, ancien ministre, par J.-M. Apathie, RTL Matin, 9 septembre 2008

(7) JMA 19 - vous aurez face à vous demain des adversaires très résolus François Bayrou en fait partie / il a dit récemment (...)

Tout aussi fréquemment, le journaliste rapporte les paroles d'un membre non d'un autre mais du même parti que l’interviewé (8), sans pour autant s’éloigner de sa stratégie contrediscursive :

Interview de J-F. Copé, ministre, par O. Galzi, Les quatre vérités, France2, 9 décembre 2008

(8) OG11 - enfin y a y a aussi y a aussi des députés de droite qui grognent un petit peu // François Bargouin par exemple chez vous dit « cette réforme de la gouvernance de France télévision/ nommer le président de France télévision / le faire nommer par le président de la république / c'est contre la démocratie / enfin c’est presque un recul démocratique » et il est UMP

Dans ce cas, la cible visée est un autre lieu commun du discours politique : l'unité du groupe d'appartenance, censé parler d'une seule voix. L’intervieweur questionne cet idéal monophonique de l'accord en lui opposant la réalité polyphonique, ou plutôt cacophonique («des députés de droite qui grognent») du désaccord. On notera notamment, en (8), l'insistance avec laquelle est explicitée l'identité politique de l'énonciateur: «François Bargouin par exemple chez vous dit [....] et il est UMP ». Façon de poser une contradiction dans les faits : la conjonction de coordination et permet de rapprocher des éléments pour mieux faire ressortir leur opposition.

Plus incisive encore : la question porte sur un propos, difficilement justifiable au regard des valeurs de la République, d’un membre du même parti : 
(9) RS1 - hier Gérard Longuet le président du groupe UMP au Sénat a crée un véritable tollé en disant que le socialiste Malek Boutih était mal placé pour diriger la Halde l’organisme qui vérifie la discrimination l'absence de discrimination en tout cas dans la société française / est-ce que Gérard Longuet a fait un dérapage raciste ?

Ce type de question enferme l'interviewé dans un détroit argumentatif : sa réponse devra se frayer un chemin entre le Charybde de la justification de l'injustifiable, au nom de la solidarité du groupe, et le Scylla de la condamnation au nom des valeurs républicaines, qui peut apparaître comme un lâchage.

La parole rapportée peut enfin être celle non d'un membre d'un autre ou du même parti que l'interviewé, mais de l’interviewé lui-même, et ce avec pareille visée contrediscursive :

Interview de J-F. Copé par O. Galzi, Les quatre vérités, France2, 9 décembre 2008

(10) OG31 - un mot d'humour pour terminer / dans la présélection du press club / pour le prix de l'humour politique / vous avez été sélectionné pour une petite phrase / à Nicolas Sarkozy vous lui auriez dit « si tu donnes les clés du parti à Xavier Bertrand / pense à faire un double » / c’était juste pour le bon mot ou vous le pensez vraiment?

Dans ce cas, la cible visée est une autre image d’Epinal passablement écornée : celle du parti politique fait de gentils membres qui n'entretiennent entre eux que des relations amicales. Le propos rapporté en (10) relève de la médisance (tempérée par sa catégorisation en «mot d'humour ») : il donne à voir, sous l'écran de la franche camaraderie et des gentils copains, la réalité de la rivalité féroce qui passe par la pratique du dénigrement.

On le voit donc : la variété des énonciateurs convoqués - autre ou même politique de l’interviewé, interviewé lui-même -, sert non à varier le type d’acte de langage réalisé par le jugement rapporté mais à confirmer sa dimension incisive en variant ses formes.

(ii) Cette « déclinaison » du jugement critique cité, que nous venons de saisir au niveau des énonciateurs, se voit confirmée par la forme que prend parfois le discours rapporté. Les occurrences analysées jusqu'à présent se construisaient autour de la parole d’un seul énonciateur. On trouve quelques exemples dans lesquels c'est non une seule mais plusieurs paroles du groupe d’appartenance politique de l’interviewé qui sont mentionnées :

Interview de X. Bertrand par R. Sicard, Les quatre vérités, France2, 2 décembre 2008

(11) RS20 - sur la politique / il y a une polémique autour de l'interpellation de l'ancien directeur de Libération / euh Rachida Dati trouve qu’il y a rien à redire / Christine Albanel est émue / Nicolas Sarkozy demande des explications // quelle est votre position là-dessus ?

Si trois discours sont rapportés - discours indirect de Rachida Dati, discours narrativisés de Ch. Albanel et de N. Sarkozy - c'est à seule fin de pointer les divergences d'opinion qui règnent à l'intérieur de l'UMP, parti de l'interviewé, divergences catégorisées comme polémique par l'intervieweur. Cette structuration du jugement rapporté en interaction conflictuelle est plus appuyée encore lorsque les voix sont organisées conversationnellement en deux tours de parole structurés par une relation de dissensus et que l'un de ces tours est le fait de l'interviewé lui-même : 
(12) JMA11 - Le Figaro de ce matin raconte que vous Luc Chatel / nouveau ministre de l'éducation / vous avez dit hier en compagnie des autres membres du gouvernement / vous avez dit que vous auriez voulu profiter / vous voudriez profiter de cet emprunt pour rénover les écoles / et le premier ministre vous a dit « pas question!»

L'acte initiatif de l'interviewé - sa proposition de faire servir le nouvel emprunt à la rénovation des écoles - se voit invalidé frontalement par la réponse négative que le premier ministre lui fait, réponse dont la forme linguistique : «pas question ! » accroît fortement la dimension polémique de toute négation.

Il apparaît donc que la médiation énonciative, dans ses différentes façons de se signifier, a fortement partie liée avec la dimension contrediscursive du propos rapporté : elle l'appuie et la met en forme. A contrario : les questions non médiées sont rarement intrusives... et les questions intrusives sont tout aussi peu souvent non médiées.

3. L’importance de ces QCM se mesure à leur nombre, ainsi qu'à leur place : sur un total de 262 questions, 29\% allient contrediscursivité et médiation énonciative. 80\% des interviews commencent, après les salutations rituelles, par ce type de question : c'est le cas de (3) et (9). De plus, en cours d'interview, l'ouverture d'un nouveau thème (que signale explicitement la mention " autre sujet » en (13)) - place à la fois sensible et structurante - se fait presque toujours (90\%) par une QCM :

Interview de J-F. Copé par O. Galzi, Les quatre vérités, France2, 9 décembre 2008

(13) OG24 - autre sujet / le sénateur Philippe Marini qui fait décidément beaucoup parler de lui en ce moment propose de supprimer la demi-part fiscale accordée aux parents isolés quand ils n’ont plus leur enfant à charge / vous en pensez quoi ?

Notons enfin que certaines questions sans médiation énonciative constituent de fait des reprises des QCM antérieures auxquelles l’interviewé n’a pas répondues (cf. infra (15)).

Comment expliquer la place structurante de ce type de question qui allie contrediscursivité et médiation énonciative?

\section{Interview politique et question contrediscursive médiée : explication}

On pourrait rendre compte de la forte présence de la médiation énonciative par le rôle discursif que tient le journaliste dans l'interaction asymétrique de l'interview : nous l'avons dit, il pose des questions. Et ces questions, il ne les pose pas à titre personnel, mais parce qu'elles se posent. Le journaliste aurait recours à des dits antérieurs parce que son rôle est celui d'un médiateur, d'un porte-parole au sens littéral : le relais de questions que se posent les Français ou qu'ils ont envie de poser.

Cette explication a sa part de vérité ; elle ne nous paraît cependant pas déterminante pour deux raisons : tout d'abord, si tel était le cas, la médiation énonciative devrait accompagner indifféremment toutes les questions, que leur teneur soit consensuelle ou dissensuelle. Or 
nous l'avons dit, ce n'est pas le cas: la médiation se porte préférentiellement, voire exclusivement, sur les questions qui introduisent un grain de sable... voire un caillou dans la machine bien huilée du discours politique. D’autre part, comme nous l'avons vu, les énonciateurs de ces discours tendent à référer à des personnes bien précises plutôt qu’à un indéfini (on dit que) ou à un ensemble comme les Français.

Les raisons de l'association de la médiation énonciative et de la dimension contrediscursive nous semblent bien plutôt résider dans les représentations actuelles du discours politique comme dans les attentes que les auditeurs et téléspectateurs ont à l'égard des médias.

(i) Le discours politique passe de plus en plus pour un écran mensonger dont la fonction principale est de légitimer le pouvoir de ceux qui le tiennent (Charaudeau 2005). Cette représentation négative se cristallise dans le syntagme langue de bois. Le journaliste se voit alors investi d'un rôle de... bûcheron, pour poursuivre la métaphore : ses questions doivent être autant de coins enfoncés dans l'épaisse écorce du discours politique ; les jugements critiques antérieurs sont autant de fentes dans ladite écorce qu'il se doit d'exploiter... Faute de quoi, il encourt le reproche de complaisance, voire de connivence, ou pire : d'être à la solde de l'homme politique qu'il interviewe.

(ii) Les téléspectateurs veulent du spectacle : que les hommes politiques soient soumis à la question, certes seulement verbale, voilà qui plaît. Un match de football est agréable à regarder pour autant qu'il y a des mouvements d'attaque, de défense, de contre-attaque. L'interview tient de la confrontation sportive ; la question contrediscursive fonctionne comme une attaque : le journaliste pénètre le terrain discursif de l'interviewé, qui se voit contraint à se défendre de cette offensive... sans commettre trop de fautes.

L’intervieweur remplit bien son rôle - au double regard de l'éthique journalistique, et des exigences télévisuelles de l'audimat - s'il pose les questions qui font mal. Notons que ces deux raisons qui poussent à interroger sur des points sensibles tendent à contrevenir aux règles de la politesse conversationnelle (Goffman 1973, Brown et Levinson 1987), qui veulent que le locuteur ménage la face (positive) de l’interlocuteur et qu'il ne pénètre pas son territoire, sa face négative. Les QCM sont autant de face threatening acts qui, dans le cadre du genre de l'interview politique, sont parfaitement bienvenues, et même attendues par les téléspectateurs.

La médiation énonciative joue un rôle complexe par rapport à cette exigence générique de questions intrusives, dans la mesure où elle fonctionne comme (i) diésation et (ii) bémolisation.

(i) En mettant le jugement intrusif au compte d'un énonciateur, le journaliste le légitime, et indirectement en alourdit la charge contrediscursive : ce n'est pas lui qui l'invente mais une personnalité de la sphère politique (ou politico-syndicale) - un énonciateur de poids - qui l'a dit. Ce fonctionnement par autorité citée se voit confirmé par le fait suivant : le nom de la personnalité mentionnée est souvent suivi d'une apposition qui explicite ses fonctions ou ses titres, comme supra en (2): «Laurence Parisot la présidente du MEDEF », ou en (5) 
«François Hollande premier secrétaire du parti socialiste ». Ces appositions au nom des énonciateurs rapportés ne sauraient être (seulement) informatifs : tout téléspectateur sait que L. Parisot est la présidente du MEDEF, que F. Hollande est le premier secrétaire du PS, etc. Elles ont pour fonction essentielle de renforcer la contrediscursivité du jugement déjà diésé par la médiation énonciative.

(ii) La médiation énonciative, si elle permet de diéser le caractère offensif du jugement contrediscursif en le légitimant, permet également de bémoliser la responsabilité énonciative du journaliste, qui se présente seulement comme le locuteur et non comme l'énonciateur du propos intrusif. Elle fonctionne, de ce point de vue, comme un bouclier défensif qui autorise à poser des questions délicates parce qu'il met par avance le locuteur à l'abri des réponses agressives : c'est pas moi qui le dit, c'est $x .$. Et par cette dimension, elle contribue d'ailleurs à favoriser la dimension contrediscursive : le journaliste peut d'autant plus appuyer sur la pédale offensive que la médiation énonciative tend à fonctionner, pour l'intervieweur, comme un paratonnerre qui écarte les possibles foudres de l’interviewé.

Cette explication nous semble corroborée par les deux faits suivants : comme nous l'avons dit, ce sont préférentiellement les questions infirmatives qui sont médiées, les questions confirmatives faisant rarement appel à ce fonctionnement énonciatif. Et d'autre part, les premières, lorsqu'elles n’ont pas recours à la médiation, ce qui est rare, s'entourent de modalisations qui sont autant d'adoucisseurs de l'intrusion, comme p. ex. dans (14) :

Interview de B. Hamon, porte-parole du PS, par F. Laborde, Les quatre vérités, France2, 15 décembre 2008

(14) FL30 - demain euh :: c’est théoriquement le le vote définitif de la réforme de l'audiovisuel public / euh finalement le la campagne si je puis dire / qu'a qu'a tenté de mener le parti socialiste pour modifier cette cette loi / n’a pas été euh extrêmement productive

L’évaluation négative portée sur une initiative du PS, non rapportée à une source externe, est celle de la journaliste : on soulignera les modalisations précautionneuses qui adoucissent par avance l'intrusion : "finalement» (appel au consensus), «si je puis dire » (demande d'autorisation) ; ainsi que la multiplication des bégaiements d'actualisation ( «le le vote », «le la campagne (...) qu'a qu'a tenté de mener ») et des pauses pleines (« demain euh :: (...)», « euh finalement « n’a pas été euh extrêmement productive »). Précisons que dans cette même interview, les questions incisives, lorsqu'elles sont médiées, ne font pas apparaître ces marques d'adoucissement.

La structure des QCM, qui associe médiation énonciative et contrediscursivité, nous semble être la réponse trouvée par les journalistes à l'attente des téléspectateurs comme du système médiatique à l'égard du discours politique : de tenter de s'introduire, à moindres frais, dans le plein qu'il exhibe à partir des failles qu'il tente de masquer.

\section{Interview, QCM et polémique}

Comment les hommes politiques répondent-ils à ces QMC ? Comment gèrent-ils leur charge offensive et la médiation énonciative qui tout à la fois la dièse et la bémolise ? L’une 
d'entre nous, dans une récente communication (Nowakowska 2010), a traité la façon dont les interviewés prenaient en compte ou ignoraient ladite médiation, notamment pour neutraliser la dimension intrusive de ce type de question. Dans le présent article, nous nous attacherons à analyser le rapport entre QCM et polémique. Notre hypothèse est que la structure de l'interview, à laquelle s'ajoute la médiation énonciative de la question, tend à préserver l'interaction de la polémique (3.1.), même si elle ne saurait empêcher qu'elle y verse parfois (3.2.).

3.1. La polémique, qu’on l'aborde en termes sémiotiques (Greimas 1979) ou pragmatiques (Burger 2010), présuppose que les interactants sont des pairs, qui entrent en concurrence pour la possession d'un même objet (modèle sémiotique), ou pour imposer leur point de vue lors d'un désaccord dans la phase de ratification d'une négociation (modèle pragmatique).

Si l'on considère les QCM à la lumière de ces définitions, il semble bien que, quelle que soit leur charge intrusive, elles ne devraient pas engendrer de polémique entre l'intervieweur et l'interviewé ${ }^{3}$ (i) pour des raisons structurales, et également (ii) pour des raisons de communication médiatique.

(i) Les interactants de l'interview ne sont pas des pairs, comme le signale leur différence de statuts : journaliste / homme ou femme politique, mais des complémentaires, comme l'indique leur différence de rôle discursif : poser des questions / répondre aux questions posées. Ce qui exclut en principe la relation de concurrence (approche sémiotique), comme la phase de désaccord (approche pragmatique), définitoires de la polémique. Reprenons une question particulièrement incisive comme :

Interview de X. Bertrand par R. Sicard, Les quatre vérités, France2, 2 décembre 2008

(2) RS13 - sur le désintérêt de cette élection / Laurence Parisot la présidente de MDF a une explication / elle dit que le responsable c'est vous / parce que la campagne a été organisée dans la plus grande pagaille

L'acte de langage direct qu'elle réalise n'est pas une accusation ad hominem - ce qui serait le cas de l'affirmation fortement polémique que l'on pourrait avoir dans un débat entre pairs :

(2’) - c’est vous le responsable du désintérêt de cette élection / parce que la campagne a été organisée dans la plus grande pagaille

- mais une question médiée: le journaliste n’accuse pas lui-même, il rapporte une accusation ; et d'autre part, cette accusation rapportée est mise en débat par la modalité interrogative qui la sous-tend : il est demandé à l'interviewé de la ratifier ou de l'invalider argumentativement. Formellement au moins donc, dans l'interview, les interactants ne sauraient (se) combattre puisqu'ils ne sont pas au même niveau, ce qui se manifeste par le fait que l'acte de langage prototypique de question, qui plus est doublé de la médiation énonciative dans les QCM, n’instaure pas une relation polémique.

\footnotetext{
${ }^{3}$ On n’abordera pas ici - dans la mesure où n'est traitée que l'interaction intervieweur-interviewé - le fait que le jugement rapporté, par sa contrediscursivité, peut être une invite faite à l'interviewé d’entrer en polémique avec l'énonciateur dudit jugement.
} 
(ii) Au niveau médiatique, les interactants ont tout à perdre de verser dans la polémique : le journaliste, parce qu'il apparaîtrait comme sortant de sa neutralité de questionneur porteparole ; l'interviewé(e), parce qu'il donnerait l'image d'une personne hyper-susceptible qui voit des accusations là où il n'y a que des questions qui se posent, qui attaque un journaliste alors qu'il ne fait que son métier...

Structuralement comme médiatiquement donc, les QCM, aussi percutantes soient-elles, n’impliquent pas la polémique. Et effectivement, l'analyse de la façon dont les interviewés leur répondent (Nowakowska 2010) fait apparaître qu'ils usent de différentes stratégies argumentatives pour désamorcer la charge intrusive de ces questions sans verser dans le conflit interactif.

3.2. Et pourtant... il arrive parfois que les QCM engendrent la polémique. Ce qu'il nous faut maintenant considérer. Nous venons de dire que la structure question (médiée) / réponse tendait à placer l'interaction hors polémique, dans la mesure où, n'étant pas des pairs, les interactants ne sauraient entrer en conflit interactif puisqu'ils n'ont pas à s'accorder sur une proposition. C’était ne pas prendre en compte l'accord implicite préalable sur lequel repose l'interaction de l'interview : le droit pour l'intervieweur de poser des questions ; le devoir, pour l'interviewé d'y répondre. Que cet accord soit remis en cause, et c’est la polémique. On en distinguera deux formes : la première porte sur le devoir de l’interviewé ; la seconde, sur le droit de l'intervieweur.

\section{Réponse « à côté ", répétition de la question et désaccord polémique}

L’interviewé doit répondre aux questions qui lui sont posées. Qu’advient-il lorsque, face à une QCM particulièrement embarrassante, l’homme politique répond... «à côté »? Le journaliste peut choisir de ne pas relever cette entorse à l'accord interactif préalable ; il peut aussi, puisqu'il ne lui a pas été répondu, réitérer sa question, ce qui ne va pas parfois sans polémique :

Début de l'interview de L. Chatel, nouveau ministre de l'éducation et porte parole du gouvernement, par J.-M. Apathie, RTL Matin, 29 juin 2009

(15) JMA1 - emprunt Sarkozy vous en avez parlé hier à Matignon où le gouvernement était réuni au grand complet / question du journal Libération à la une ce matin (JMA montre la une de Libération à L. Chatel) « Emprunt Sarkozy : La France est-elle en faillite ? »

LCH2 - écoutez c’est quand même légitime que après le discours du président de la république du congrès ::: / après le remaniement du gouvernement / le premier ministre réunisse l'ensemble de son gouvernement pour euh mettre en perspective la feuille de route du président parmi laquelle figure effectivement la mise en place d'un grand emprunt pour investir dans les secteurs de ce qu'on appelle de sortie de crise

JMA3 - parce qu'il n’y a plus de sous dans les caisses ? parce que la France est en faillite ? dit Libération

LCH4 - non / vous savez le gouvernement il est sur trois fronts en même temps / le premier front, c'est la riposte à la crise / ça c’est le plan de relance (...) / le deuxième front euh c’est ::: la réponse c’est la 
maîtrise du déficit / et la poursuite des reformes/ Alain Duhamel l'a dit tout à l'heure/ c'est pas parce qu'il y a la crise qu'il faut pas continuer à réformer le pays (...) le troisième front / c'est celui d'investissement dans les secteurs d'avenir (...) / la France elle doit miser sur les secteurs où elle a un avantage compétitif qui pourront dans les années qui viennent créer des emplois et de la croissance et c'est l'objet précisément de ce grand emprunt

JMA5 - (pointant l'index en direction de l'interviewé) et alors la France est-elle en faillite oui ou non ?

LCH6 - la France n'est pas en faillite parce qu'un état n'est pas en faillite Jean-Michel Apathie...

JMA7 - elle en a de la chance !

LCH8 - la France elle est dans une situation budgétaire que l'on connaît tous hein à savoir que en gros les recettes couvrent environ deux tiers de nos dépenses (...)

JMA9 - de combien d'argent avez-vous besoin pour financer ces dépenses d’avenir Luc Chatel ?

Soit la QCM du journaliste, en JMA1 : «question du journal Libération à la une ce matin : Emprunt Sarkozy : La France est-elle en faillite ? ». L. Chatel (LCH2) commence par justifier ( «c’est quand même légitime ») un élément du thème général introductif, la réunion de tout le gouvernement (élément (a) du module) sur lequel ne portait pas l'ombre d'un questionnement, et termine son tour de parole sur « la mise en place d'un grand emprunt », qui reprend le thème de la question du journaliste («Emprunt Sarkozy »), mais ne répond en rien à l'interrogation qui en constituait le rhème : « La France est-elle en faillite ? »... Ce qui conduit le journaliste à répéter une première fois sa question en JMA3, puis, du fait que L. Chatel y répond très latéralement en $\mathrm{LCH} 4$, à la réitérer une troisième fois en JMA5. De la première à la seconde et surtout de la seconde à la troisième occurrence de ladite question, la contrediscursivité se transforme en désaccord polémique : par la réitération, l’intervieweur signifie à son interlocuteur qu'il n’accepte pas comme réponses valables les propos que celuici lui a présentés comme tels (LCH2, puis LCH4), ce qui constitue autant de rebuffades pour l'interviewé. Le glissement vers le conflit se manifeste, pour ce qui est de la parole du journaliste, d'une répétition à l'autre, par la montée d'intonations d'agacement ; et, lors de la troisième occurrence (JMA5), par plusieurs éléments : geste de l'index, qui semble acculer l'interlocuteur à répondre ; effacement de la médiation énonciative, ce qui revient à mettre directement en contact frontal la parole de l'intervieweur et celle de l'interviewé; renforcement introduit dans l'interrogation par les adverbes responsifs oui ou non. A l'interrogation ouvertement polémique au quadruple niveau intonatif, gestuel, énonciatif et syntaxique de JMA5, l’homme politique fait, en LCH6, une réponse toute aussi polémique, au double niveau argumentatif et rhétorique : «la France n’est pas en faillite parce qu'un état n’est pas en faillite Jean-Michel Apathie... ». Il réplique par la négative à la question posée en appuyant sa réponse (parce que) sur la négation du présupposé sur lequel elle reposait («un état n'est pas en faillite »), ce qui revient à invalider la pertinence de ladite question; et interpelle l'intervieweur par l'apostrophe de ses prénom et nom («Jean-Michel Apathie »), figure dont C. Kerbrat-Orecchioni (2005) a décrit le fonctionnement tendanciellement agonal en interaction verbale. Le journaliste en JMA7 use d'une pause respiratoire de l'interviewé pour glisser une évaluation positive ironiquement antiphrastique : « elle en a de la chance !». L'interaction est ici ouvertement conflictuelle : les interactants jouent qui à saper les bases du discours de l'autre, qui à le moquer... Cependant, après cette passe d'armes réciproque, 
intervieweur et interviewé décident tacitement de retrouver la politesse de l'entretien un instant menacée : L. Chatel enchaîne par une réponse technique (LCH8), que semble cette fois-ci valider le journaliste, puisque, au lieu de répéter sa question initiale, il va poser à la suite (JMA9) une autre question...: « de combien d'argent avez-vous besoin pour financer ces dépenses d'avenir Luc Chatel ? », dans laquelle seule l'apostrophe « Luc Chatel » introduit encore une petite touche dissensuelle...

La polémique, dans ce premier type de cas, procède de ce que l’interviewé transgresse la règle implicite du contrat de l’interview selon laquelle il doit répondre aux questions qui lui sont posées.

\section{Contestation de la légitimité de la médiation énonciative et polémique}

Nous avons explicité la façon dont la médiation énonciative permettait la formulation du jugement intrusif. La contestation de la légitimité de l'énonciateur rapporté comme autorité citée introduit de la polémique :

L'avant-veille d'une journée de grève unitaire, au cours du JT de $20 \mathrm{H}$ de France 2, D. Pujadas interviewe F. Fillon, premier ministre. L'entretien est précédé d'un reportage auprès de la population qui exprime son ressentiment à l'égard d'un gouvernement qui refuse d'augmenter les salaires alors qu'il vient d'accorder aux banques un prêt important pour les sauver de la faillite

(16) DP5 - vous l'avez entendu monsieur le premier ministre y a un sentiment pour beaucoup d'injustice /des milliards donnés aux banques qui sont considérées à tort ou à raison comme parties prenantes de la crise financière / et rien ou peu pour les Français pour la consommation pour le pouvoir d’achat

FF6 - y a un moment où il faut que chacun prenne ses responsabilités et je dirais y compris le système médiatique / on peut pas dire "à tort ou à raison donnés aux banques » / pardonnez-moi mais je sais bien que c'est le discours qui est tenu comme ça par tout le monde

DP7 c'est un sentiment qu'on entend et qu'on vient encore d'entendre...

FF8 - oui mais c'est pas une question de sentiment / qu'est-ce que diraient les Français si en octobre ils s'étaient présentés au guichet du Crédit Agricole du Crédit Lyonnais ou de la BNP et qu’on leur avait dit « Le guichet est fermé vos économies se sont envolées » / parce que c’est ça la situation face à laquelle le gouvernement s'est trouvé et donc ce que nous avons fait c’est sauver d’abord les économies des Français (...)

La QCM en DP5 porte sur le prêt important accordé aux banques, catégorisé comme « injustice » au travers de la médiation énonciative " $\mathrm{y}$ a un sentiment pour beaucoup ». L’interviewé, au lieu de répondre à la question que pose ce jugement, commence par en appeler à la «responsabilité » du «système médiatique » auquel est refusé le droit de rapporter certains discours implicitement posés comme fallacieux, fussent-ils «tenu(s) par tout le monde ». La remontrance, même si elle s'accompagne de mitigation - indirection de l'admonestation (remplacement du vous accusateur par des indéfinis (chacun, on) et dissolution dans une entité plus large («le système médiatique ») qui évitent l’attaque ad hominem, modalisation du dire («je dirais »), demande d'excuse («pardonnez-moi ») - est fortement polémique puisqu'elle met en cause la compétence, voire l’éthique du journaliste, 
coupable de rapporter des propos sans fondement. Celui-ci semble piqué au vif : notons le chevauchement de DP7 sur la fin du tour FF6, qui signale la conflictualité. Il tente de légitimer à nouveau sa question et sa médiation en corrigeant le terme de discours proposé par F. Fillon ( « c'est le discours qui est tenu ») par celui de sentiment (« c'est un sentiment qu'on entend »), qu’il avait précédemment employé en DP5, et qui lui paraît de plus de poids. Peine perdue : FF8 nie catégoriquement la pertinence justificative de ce terme («c’est pas une question de sentiment /»), et poursuit par une question rhétorique faisant judicieusement appel à la parole - imaginée - des Français (« qu’est-ce que diraient les Français si en octobre ils s’étaient présentés au guichet du Crédit Agricole (...) », c’est-à-dire au même type de médiation énonciative que l’interviewé. L’homme politique a gagné : il a invalidé la pertinence de la médiation énonciative qui justifiait la question de l'intervieweur et du même coup la pertinence de ladite question ; il va pouvoir se permettre de ne pas y répondre.

La polémique, ici relativement feutrée et polie, engendrée par la mise en cause de la médiation énonciative de la question de l'intervieweur, nous allons la retrouver mais exacerbée et grossière dans le fragment d'interview suivant :

Interview par J.-M. Apathie de B. Tapie, RTL Matin, 9 septembre 2008. Cet ancien ministre, homme d'affaires, s'est vu accorder par un tribunal arbitral 285 millions d'euros d'indemnité dans le dossier Adidas qui l'a opposé durant 15 ans au Crédit Lyonnais. Il sera auditionné le lendemain de l'entretien par les députés.

(7) JMA19 - vous aurez face à vous demain des adversaires très résolus Français Bayrou en fait partie / il a beaucoup critiqué cette sentence arbitrale et il a dit récemment « voilà un état qui n’a plus un sou dans ses caisses et qui donne le moyen à monsieur Tapie de rembourser ses dettes aux frais du contribuable »

BT20 - vous voulez pas en dire d'autres ? parce qu'il en a dit quelques-unes des conneries

JMA21 - il en a dit beaucoup...

BT22 - ouais ce que je voudrais vous dire c’est que je suis épaté moi / de l'écart qu’il y a entre l'importance que vous donnez à ce monsieur / où tous les jours on fait les commentaires de ce qu'il pense ce qu'il dit des bons points qu'il donne des mauvais points qu'il donne / alors que politiquement il incarne plus rien (...)

JMA23 - mais sur le fond ? il dit « l'état n'a plus de sous...

BT24 non mais sur le fond je suis heureux qu'il vienne demain pour tout vous dire

JMA25 - et il va / et il va donner plusieurs centaines de millions xx à Bernard Tapie »

BT26 - oui mais il va me le dire il va me le dire je l'espère demain quand je serai là / (...) je m’attends à ce qu'un jour il déclare / « c'est un scandale qu’on meure encore du cancer » / et vous avez des gens qui diront « oui c'est vrai putain c'est quand même dommage qu'on meure d'un cancer » / (...)

Sans entrer dans le détail de cette plage, notons qu'à la QCM du journaliste en JMA19, B. Tapie (BT20) répond par une dévalorisation injurieuse («connerie ») du discours de l'énonciateur rapporté, suivie en BT22 de sa disqualification en tant qu'autorité politique. Le journaliste en JMA23, après avoir fait ironiquement chorus avec B. Tapie en JMA21, ne discute pas de cette récusation de la médiation énonciative de sa question mais tente d'en répéter le jugement intrusif, auquel il souhaite que l'interviewé réponde : « mais sur le fond ? il dit «l'état n'a plus de sous... ». Il est interrompu par B. Tapie qui très polémiquement 
s'inscrit dans la parole de l'intervieweur en reprenant le syntagme « sur le fond », pour mieux la détourner: "non mais sur le fond je suis heureux qu'il vienne demain pour tout vous dire ». Et au lieu de répondre à la question de l’intervieweur, il renouvellera satiriquement sa critique de l'énonciateur Bayrou et de son discours, ce qui indirectement invalide l'autorité du journaliste à poser des questions. La polémique, qui dans les deux précédentes interviews analysées, avait réussi à être désamorcée, va ici se poursuivre, plus ou moins ouvertement, jusqu’à éclater :

(18) BT68 - non mais je trouve scandaleux ce que vous faites vous essayez...

JMA69 - mais c'est pas scandaleux...

BT70 - c'est un scandale...

JMA71 - poser des questions ce n'est jamais un scandale Bernard Tapie !

BT72 - non c'est pas ça poser des questions pour laisser croire à ceux qui vous entendent que le fait d'avoir de toucher des honoraires rend la sentence suspecte /et voilà je suis pas plus con que vous je comprends ce que vous voulez faire

Indignation, imputation de sous-entendus, grossièretés : c’est, au-delà de la remise en cause de l'autorité du journaliste à poser des questions, la possibilité de la poursuite de l'interview qui est atteinte. Et d'ailleurs, cette menace devient réalité quelques tours de paroles plus loin :

(19) JMA89 - vous aurez votre fiche sur Edwige?

BT90 - mais ça j'en ai rien à foutre j'ai rien à cacher / ça fait exactement 25 ans que vous me regardez dans le ::::: pour savoir si j'ai un polype / alors vous savez moi je $\operatorname{xxxx}$ (il se détourne de l'interaction et fait mine de se lever, ce qu'il fait... mettant de la sorte fin à l'interaction)

JMA91 - (rire) Bernard Tapie on voit en tout cas sur quel ton il répondra demain aux questions de l'assemblée nationale

A une question particulièrement intrusive (JMA89), à la limite de la provocation, et que l'intervieweur, contrairement à sa stratégie jusqu'alors, ne prend pas la peine de médier énonciativement, l'interviewé répond par une scatologie carnavalesque... avant de mettre fin à l'interaction. Et le combat cessa faute de combattants...

Les QCM peuvent virer à la polémique : si les interactants passent outre les droits et devoirs qui fondent le contrat tacite de l'interview.

\section{Conclusion}

Nous avons pris pour objet dans cette étude un élément de l'interview politique (brève) sur lequel l'attention des chercheurs, à notre connaissance, ne s'était pas focalisée: les questions contrediscursives médiées, qui permettent au journaliste d'être d'autant plus incisif dans son questionnement qu'elles se présentent comme rapportant un discours antérieur 
autorisé. Nous avons décrit cette association, puis en avons proposé une explication, avant de nous interroger sur son potentiel polémique. Les QCM, du fait de la structure asymétrique de l'interaction de l'interview et en appui sur la médiation énonciative, permettent de flirter avec la polémique sans la consommer, sans se consumer à son feu... à moins que ne soient remis en cause les fondements contractuels de ce genre discursif : que l'interviewé a le devoir de répondre aux questions que l’intervieweur a le droit de lui poser.

Il s’agit là d'un premier travail. Il conviendra à l'avenir de se pencher sur l'histoire de l'interview politique pour tâcher de dater l'introduction de ce type de question et surtout sa généralisation, de voir également ses liens éventuels avec le format - brièveté - du sous-genre dans lequel nous l'avons analysé et le type de l'émission qui l'accueille ; de mettre en relation précise les QCM avec l'histoire du discours politique et de ses représentations, ainsi qu'avec l'histoire des médias. Nous envisageons également de comparer l'usage que les différents journalistes font des QCM, qui détermine leur style en tant qu'intervieweurs ainsi que celui de l'émission qu'ils animent : il apparaît p. ex. que J.-.M. Apathie en use sans modération le matin sur RTL, ce qui lui vaut l'opinion d'un intervieweur redoutable et à la matinale de RTL une audience respectable, alors que les différents intervieweurs de l'émission Les quatre vérités sur France 2 en font un usage plus modéré, ce qui confère à l'émission une tonalité plus posée, moins conflictuelle.

Enfin un travail contrastif sur l'usage des QCM dans l'interview politique de différents pays nous paraît pertinent : ce type de question, en tant que face threatening act potentiel, met en jeu la ligne de démarcation, mouvante d’une culture à l'autre, entre ce qui autorisé et ce qui est interdit.

\section{Références bibliographiques}

Brown P. et Levinson S., 1987, Politeness: Some universals in language usage, Cambridge: Cambridge University Press.

Burger M., 2004, « Média Debates : the Discourse of the Host in a TV Talk-Show », in Carlos Gouveia, Carmida Silvestre \& Lu'sa Azuaga (eds), Discourse, Communication and the Entreprise : Lingusitics Perspectives, Lisbon, Ulices, 345357.

Burger M. \& Martel G., 2005, Argumentation et communication dans les médias, Québec : Nota Bene.

Burger M., 2010, « “Ce passage n’est pas z’encore à l’abri” : une caractérisation praxéologique du désaccord polémique dans la production d’informations télévisées », conférence au séminaire Praxiling, Montpellier, mars 2010.

Charaudeau P., 2005, Le discours politique. Les masques du pouvoir, Paris : Vuibert.

Clayman S. and Heritage J., 2002a, «Questioning Presidents: Journalistic deference and adversarialness in the press conferences of Eisenhower and Reagan », Journal of Communication 52 (4): 749-77.

Clayman S. and Heritage J., 2002b, The News Interview: Journalists and Public Figures on the Air, Cambridge, Cambridge University Press.

Constantin de Chanay H. \& Kerbrat -Orecchioni C., 2007, « 100 minutes pour convaincre : l'éthos en action de Nicolas Sarkozy », in M. Broth et al. (éd.) Le français parlé des médias, Stockholm, Acta Universitatis Stokhomiensis, 309-329.

Goffman E., 1973, La Présentation de soi, Paris : Minuit.

Greimas A., 1979, Sémiotique : dictionnaire raisonné de la théorie du langage, Paris : Hachette.

Kerbat-Orecchioni C., 2005, Le discours en interaction, Paris : Armand Colin. 
Nowakowska A., 2010, "Stratégies de l’interviewé pour répondre à une question médiée intrusive ", in Actes du CMLF 2010, New-Orleans, www.linguistiquefrançaise.org. 